\title{
Robotic transduodenal excision of ampullary tumour
}

\author{
Francis C. H. Wong, Eric C. H. Lai, Daniel T. M. Chung, Chung Ngai Tang \\ Department of Surgery, Pamela Youde Nethersole Eastern Hospital, Chai Wan, Hong Kong, China \\ Correspondence to: Chung Ngai Tang, MBBS, FRCS. Department of Surgery, Pamela Youde Nethersole Eastern Hospital, 3 Lok Man Road, Chai \\ Wan, Hong Kong, China. Email: tangcn@ha.org.hk.
}

\begin{abstract}
Ampullary tumours are uncommon lesions with potential risk of malignancy. The management is excision by either endoscopic ampullectomy, pancreaticoduodenectomy or transduodenal excision. Endoscopic ampullectomy offers a less invasive approach, whereas pancreaticoduodenectomy allows radical excision of the tumour. They both carry their own limitations. Transduodenal excision of ampullary tumour offers significantly lower risks with low recurrence rate, and can be offered for benign or early grade tumours. Limited cases of laparoscopic transduodenal excision of ampullary tumours were reported in the literature, probably due to the technical difficulties in performing the operation, especially during the reconstruction of the pancreaticobiliary ducts with the laparoscopic approach. In the era with robotic surgical systems, the technical demanding procedures are greatly facilitated. Hence, we are writing to report a case of transduodenal excision of ampullary tumour with the robot assisted laparoscopic approach.
\end{abstract}

Keywords: Robotic; transduodenal excision; ampulla; tumour; operative technique

Submitted Jun 21, 2016. Accepted for publication Oct 14, 2016.

doi: 10.21037/hbsn.2016.12.04

View this article at: http://dx.doi.org/10.21037/hbsn.2016.12.04

\section{Introduction}

Ampullary tumours are uncommon, with an incidence rate of $0.4-0.48$ per 100,000 in 1973-2005 (1). In recent years, with the increased availability of endoscopy and radiological imagings, the incidence of ampullary tumour is expected to rise. The management for ampullary tumours is complete excision, either through endoscopy as endoscopic ampullectomy or with surgical excision by means of pancreaticoduodenectomy or transduodenal excision. While endoscopic ampullectomy is being employed to small benign tumours, larger ampullary masses require surgical resection. Traditional pancreaticoduodenectomy allows radical excision of the tumour, but carries significant morbidity and mortality. Transduodenal excision of ampullary tumour, on the other hand, offers significantly lower risks and can still achieve low recurrence rate, for benign or early invasive tumours $(2,3)$.

In the era of minimal invasive surgery, Rosen et al. reported the first case of laparoscopic transduodenal resection of a periampullary villous adenoma in 2003 (4). To date, there were four reported cases of laparoscopic transduodenal excision of ampullary tumours in the literature (4-6), showing the feasibility of minimal invasive surgeries in performing transduodenal excision. While performing transduodenal excision of ampullary tumour, a technically demanding step lies at the fine suturing in reconstructing the pancreatic and biliary duct to the duodenum. Suboptimal suturing may lead to ductal strictures, which can be complicated with pancreatitis and cholangitis.

Nowadays, with the introduction of the robotic operation systems, the fine suturing of delicate tissues can be facilitated by the dexterity in arms movements, stable camera with 3 -dimentional views, along with surgeon comfort with ergonomic position. Hence, we are writing to report a case of robotic assisted transduodenal excision of ampullary tumour.

\section{Case report}

A 63 years old woman presented with subjective weight loss and was diagnosed to have an ampullary mass in September 


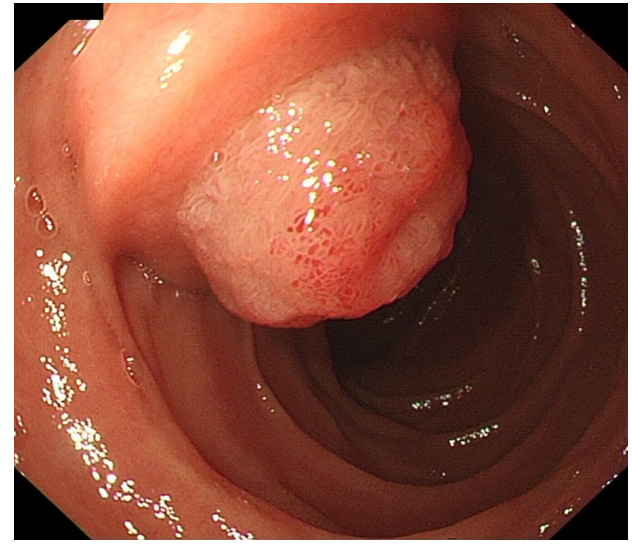

Figure 1 Oesophagogastroduodenoscopy (OGD) showing a villous growth over ampulla of Vater.

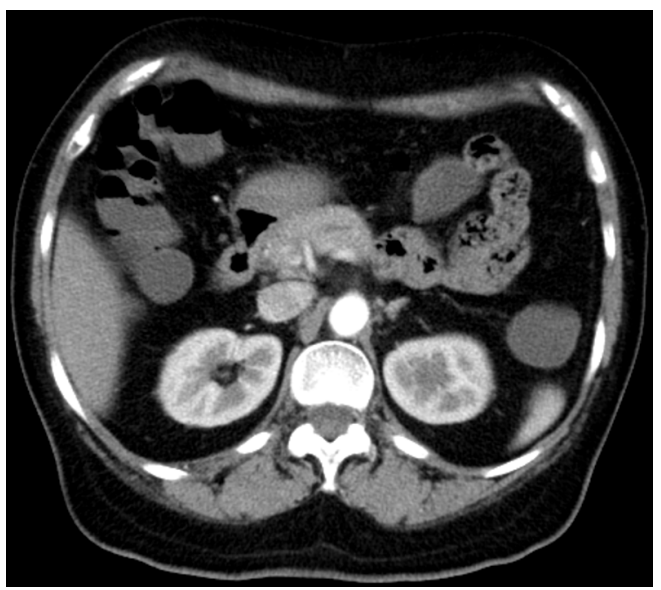

Figure 2 Contrast enhanced CT scan showing no enhancing lesion. CT, computed tomography.

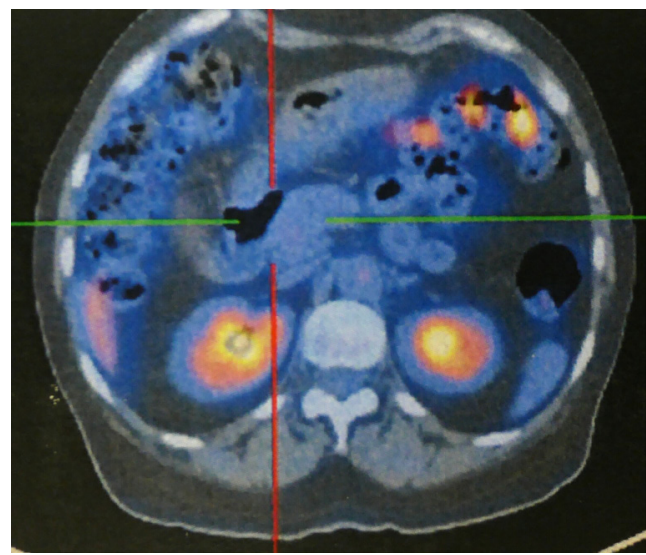

Figure 3 PET scan showing no uptake over the papillary region. PET, positron emission tomography.

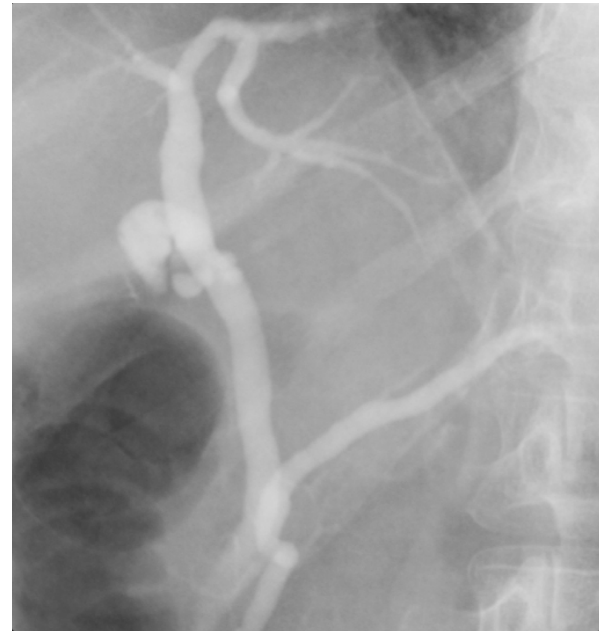

Figure 4 ERCP in 1 year post-operatively showing no biliary or pancreatic ductal strictures. ERCP, endoscopic retrograde cholangiopancreatography.

2013. Oesophagogastroduodenoscopy (OGD) showed a villous growth over the ampulla of Vater (Figure 1). Multiple biopsies were taken from the villous growth. Pathology reviewed villous adenoma with mild dysplasia. Computed tomography (CT) scan and positron emission tomography (PET) scan showed no contrast enhancing lesion and no fluorodeoxyglucose (FDG) uptake over the papillary region (Figures 2,3). Robot-assisted laparoscopic transduodenal excision of ampullary tumour was performed. Post-operative recovery was uneventful. On postoperative day 5 , water-soluble contrast meal showed no contrast extravasation. Oral diet was tolerated. Patient was discharged on post-operative day 7. Pathology of the ampullary tumour showed tubulovillous adenoma with moderate to severe dysplasia. Two follow-up endoscopic retrograde cholangiopancreatography (ERCP) were performed 4 months and 1 year after the operation, showing no recurrence of the ampullary lesion and no bile duct or pancreatic duct stricture (Figure 4). She has continued follow up at our clinic. To date, at 30 months after the operation, she remained well.

\section{Surgical technique}

Robot-assisted transduodenal excision of ampullary tumor was performed. The da Vinci S Surgical System (Intuitive Surgical Inc., Sunnyvale, CA, USA) was used. Patient was placed in supine position under general anaesthesia. Nasogastric tube and urinary catheter were inserted. 


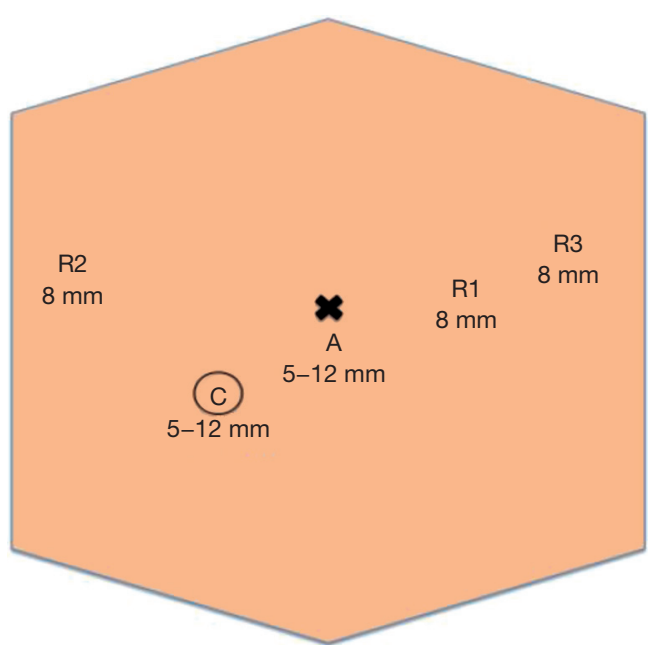

Figure 5 Robotic arms, camera and assistant port placements. C, camera port; R, robotic arm; A, assistant port.

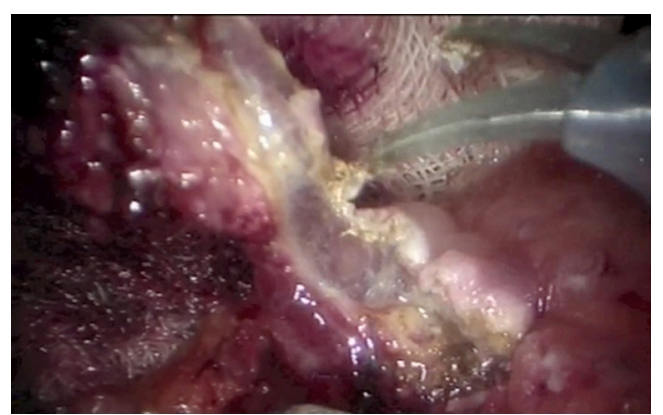

Figure 6 Excision of the ampullary tumour after raised by submucosal adrenaline saline injection.

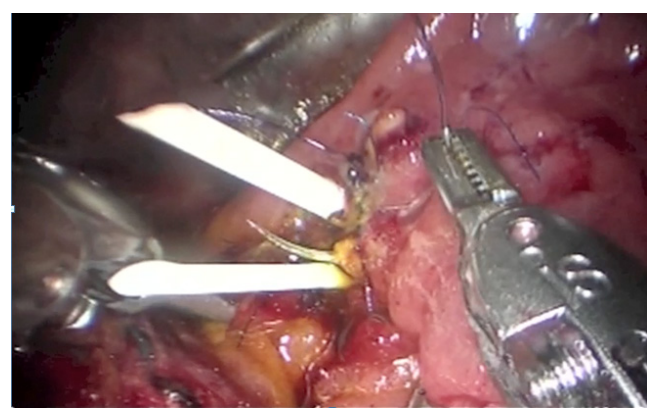

Figure 7 Insertion of stent into pancreatic and bile duct individually and anchored with 3/0 Monocryl sutures.

Subumbilical incision was made with insertion of a $12 \mathrm{~mm}$ trocar by open technique. Pneumoperitoneum was created with $\mathrm{CO}_{2}$ at pressure of $12 \mathrm{mmHg}$. Diagnostic laparoscopy was performed. Under direct visualization, the $12 \mathrm{~mm}$ camera port was inserted at right-sided abdomen, one $8 \mathrm{~mm}$ robotic port and two $8 \mathrm{~mm}$ robotic ports were inserted at right- and left-sided abdomen respectively (Figure 5). Robotic system was docked in from the head end of the bed.

Hepatic flexure of colon was mobilised. Duodenum was mobilised with Kocher maneuver. Longitudinal duodenal incision was made over the anterolateral wall of the $2^{\text {nd }}$ part of duodenum. The ampullary tumor was raised with submucosal injection of $6 \mathrm{~mL}$ of diluted adrenaline saline, and was completely excised from duodenal wall and put into specimen bag (Figure 6). Pancreatic duct and bile duct were identified and sutured back to the duodenal mucosa with interrupted 6/0 polypropylene sutures (Prolene, Ethicon, Norderstedt, Germany). Gauge 14 silicone catheters (Nutricath "S", Vygon, Ecouen, France) were inserted into the pancreatic duct and bile duct for stenting, and were anchored with 3/0 poliglecaprone 25 sutures (Monocryl, Ethicon, Somerville, NJ, USA) (Figure 7). Enterotomy at the $2^{\text {nd }}$ part of duodenum was closed with continuous 3/0 V-Loc absorbable suture (Covidien, Mansfield, Massachusetts, USA) in single layer fashion. The operative site was irrigated with warm saline, and a silicon drain was placed at right subhepatic space via the right lateral port. Robotic system was docked out. Specimen bag was retrieved via the subumbilical wound. The operative time was 250 minutes. The blood loss was $20 \mathrm{~mL}$.

\section{Discussion}

Ampullary tumour can be resected through endoscopic and surgical approaches. Surgical approaches include transduodenal excision and pancreaticoduodenectomy.

Endoscopic ampullectomy has gained popularity in the past decade with the increasing experience in interventional endoscopy, as it could avoid the need of surgical intervention for selected cases. However, the endoscopic approach is limited in cases of recurrence of inflammatory disease with sclerosis of the pancreatic sphincter or when a neoplastic lesion exceeds the size that allows safe endoscopic removal (2). Moreover, it carries $15 \%$ overall complication rate, including risk of pancreatitis, bleeding, perforation, cholangitis and papillary stenosis. Mortality risk is up to $0.3 \%$ and the risk of local recurrence was reported up to $33 \%(7,8)$. Therefore, endoscopic ampullectomy may be considered in patients with small $(<2 \mathrm{~cm})$ villous or tubulovillous adenoma, adenomyosis, neurinoma and 
chronic papillitis (9), and in patients who are poor surgical candidates.

On the other hand, pancreaticoduodenectomy allows radical excision of the tumour, but carries significant risks. Greenblatt et al. reviewed 4,945 patients who received elective pancreaticoduodenectomy from 2005 to 2009, showing a serious complications rate of $27.1 \%$ and 30 -day mortality risk of $2.6 \%$ (10). On the contrary, the surgical risk from transduodenal excision is lower. The largest series of open transduodenal ampullectomy to date is reported by Schneider et al. (2). A total of 83 cases were reviewed. Morbidity rate of $24 \%$ and mortality rate of $1.2 \%$ were reported. Of the 44 cases with adenomas, while majority of which were indicated for recurrence after endoscopic removal, the local recurrence rate after transduodenal excision was only $2.3 \%$ ( 1 in 44 cases). In view of the potential lymph node metastasis in T1 tumours, which was reported to be as high as $10-28 \%(11,12)$, that could only be removed by pancreaticoduodenectomy, transduodenal excision is reserved for earlier-staged lesions, namely, TIS or high-grade dysplasia adenomas, large adenomas, recurrent adenomas after endoscopic excision, and technically unresectable tumours by endoscopy, and for patients with high operative risk for pancreaticoduodenectomy.

Although there was fruitful development of laparoscopic equipment and skills throughout the past years, there were only four reported cases applying minimal invasive approach for transduodenal excision in the literature (4-6). The small number of cases reported could possibly be due to the procedure being technically difficult with the complexity of the ampulla anatomy and difficulties in reconstructing the pancreaticobiliary duct system (5), ergonomically unfavourable and requiring experienced surgeons in laparoscopic surgeries. Compared with the open surgery counterpart, the existing results from laparoscopic approach showed a lower blood loss (40-50 mL) and a shorter hospital stay (6-9 days), with no post-operative complications or recurrence reported (4-6).

Despite the fact that robotic surgical system is being applied to various operations, in attempt to overcome the technical difficulties encountered in traditional laparoscopic operations, robotic transduodenal resection of ampullary tumours has not been widely reported. Downs-Canner et al. reviewed 26 cases of robotic resection of benign duodenal pathologies, in which 9 cases were robotic transduodenal ampullectomy (13). However, the results were gathered collectively from ampullary and non-ampullary lesions and the cases were heterogeneous in pathology and tumour locations, the results from the nine ampullectomy cases were not broken down and analysed. Theoretically, the high degree of movements, dexterity and stable tissue retraction conveyed by the robotic arms, the 3 -dimentional magnified image of the operative field, and the stable camera view can help a lot in the most technically demanding part of the operation, namely, the re-approximation of the pancreatic duct and bile duct to the duodenal mucosa after completing resection. In the literature, so far, there is no published series for robotic transduodenal resection of ampullary tumours to demonstrate its potential advantages.

In our patient, we employed the robotic surgical system when performing transduodenal excision of the ampullary tumour. Comparing to the existing results from laparoscopic transduodenal ampullectomy, our patient had lower amount of blood loss and a shorter hospital stay. The result supports our proposition that applying robotic surgical system in transduodenal ampullectomy is advantageous for this group of patient. However, more cases are needed for analysis to elicit the potential benefits.

\section{Conclusions}

Robot-assisted transduodenal excision of ampullary tumour is a feasible and safe option for selected benign ampullary tumours.

\section{Acknowledgements}

None.

\section{Footnote}

Conflicts of Interest: The authors have no conflicts of interest to declare.

\section{References}

1. Albores-Saavedra J, Schwartz AM, Batich K, et al. Cancers of the ampulla of vater: demographics, morphology, and survival based on 5,625 cases from the SEER program. J Surg Oncol 2009;100:598-605.

2. Schneider L, Contin P, Fritz S, et al. Surgical ampullectomy: an underestimated operation in the era of endoscopy. HPB (Oxford) 2016;18:65-71.

3. Beger HG, Treitschke F, Gansauge F, et al. Tumor of the ampulla of Vater: experience with local or radical resection in 171 consecutively treated patients. Arch Surg 
1999;134:526-32.

4. Rosen M, Zuccaro G, Brody F. Laparoscopic resection of a periampullary villous adenoma. Surg Endosc 2003;17:1322-3.

5. Zhang RC, Xu XW, Wu D, et al. Laparoscopic transduodenal local resection of periampullary neuroendocrine tumor: a case report. World J Gastroenterol 2013;19:6693-8.

6. Ahn KS, Han HS, Yoon YS, et al. Laparoscopic transduodenal ampullectomy for benign ampullary tumors. J Laparoendosc Adv Surg Tech A 2010;20:59-63.

7. Espinel J, Pinedo E, Ojeda V, et al. Endoscopic ampullectomy: a technical review. Rev Esp Enferm Dig 2016;108:271-8.

8. Patel R, Varadarajulu S, Wilcox CM. Endoscopic ampullectomy: techniques and outcomes. J Clin Gastroenterol 2012;46:8-15.

Cite this article as: Wong FC, Lai EC, Chung DT, Tang CN. Robotic transduodenal excision of ampullary tumour. HepatoBiliary Surg Nutr 2017;6(5):312-316. doi: 10.21037/ hbsn.2016.12.04
9. Paramythiotis D, Kleeff J, Wirtz M, et al. Still any role for transduodenal local excision in tumors of the papilla of Vater? J Hepatobiliary Pancreat Surg 2004;11:239-44.

10. Greenblatt DY, Kelly KJ, Rajamanickam V, et al. Preoperative factors predict perioperative morbidity and mortality after pancreaticoduodenectomy. Ann Surg Oncol 2011;18:2126-35.

11. Lee H, Park JY, Kwon W, et al. Transduodenal Ampullectomy for the Treatment of Early-Stage Ampulla of Vater Cancer. World J Surg 2016;40:967-73.

12. Winter JM, Cameron JL, Olino K, et al. Clinicopathologic analysis of ampullary neoplasms in 450 patients: implications for surgical strategy and long-term prognosis. J Gastrointest Surg 2010;14:379-87.

13. Downs-Canner S, Van der Vliet WJ, Thoolen SJ, et al. Robotic surgery for benign duodenal tumors. J Gastrointest Surg 2015;19:306-12. 